\title{
The Effect of Heterogeneous Traffic Distributions on Load Balancing in Mobile Communications: An Analytical Model
}

\author{
K.-C. Chu, C.-S. Wang, W.-W. Jiang, N.-C. Hsieh
}

\author{
Kuo-Chung Chu, Wey-Wen Jiang, Nan-Chen Hsieh \\ Department of Information Management, \\ National Taipei University of Nursing and Health Sciences \\ No.365, Mingde Rd., Beitou Dist., \\ Taipei City 11219, Taiwan (R.O.C.) \\ $\{$ kcchu, jiang6, nchsie\})@ntunhs.edu.tw \\ Chun-Sheng Wang \\ Department of Information Management, \\ Jinwen University of Science and Technology \\ No.99, Anzhong Rd., Xindian Dist., \\ New Taipei City 23154, Taiwan (R.O.C.) \\ seanwang@just.edu.tw
}

\begin{abstract}
This paper investigates the load balancing problem in an environment of heterogeneous traffic distributions. An analytical model is proposed to determine the effect of heterogeneous traffic distributions on load balancing, in which a generic measure of load balancing level (LBL) that is a function of traffic type coefficient (TYC) and call blocking probability of cells is to analyze the expected level of the load balance. We consider both voice traffic and data traffic to determine which kind of traffic has the greater effect. The performance of cellular systems with sectorization is evaluated; they are normal case $(\mathrm{N})$ of homogeneous distribution and linear case $(\mathrm{L})$ of heterogeneous distribution. The analysis results indicate that the TYC has a significant effect on the accommodation capacity, in which voice calls outperform data calls because the LBL can easily distinguish between normal and linear distributions. Load balancing can be achieved more easily for voice only traffic than for data only traffic. Sectorization is more effective in achieving load balancing in the scenario of the heavier loads than in the lighter loads. The paper results are useful for network planning to optimize the channel allocation for different traffic type's distribution.
\end{abstract}

Keywords: analytical model, heterogeneous distributions, load balancing, mobile communications, QoS.

\section{Introduction}

Several studies have evaluated the capacity of mobile cellular systems, but most of them (e.g., $[1,2])$ assume a homogeneous spatial traffic distribution, as it best fits the system's characteristics to have all signals share all the spectral resources However, homogeneous traffic distribution among base station (BS)/sectors/cells (equal cell loads) is very uncommon in practice. Even though sufficient capacity is planned in a cellular system, heterogeneous traffic distribution may occur in other cells, creating a "hot spot" that exceeds the pre-determined capacity and introduces a large blocking probability, as the quality of service (QoS) of such cells may be degraded, especially below a pre-defined threshold. This is why load balancing is the most important issue to be discussed before network planning in terms of optimal resource allocation. 
In [3], Ning et al. discuss load balancing by using a hybrid scheme of channel borrowing scheme and load transfer, it allows borrowing channels from light load cells, and ongoing calls can be transferred from heavy load cells into the overlapping cells they are light load. To improve global resource utilization and reduce regional congestion given heterogeneous arrivals, [4] requires load balancing among multiple cells. However, their works cannot be applied to general system because a lot of issues are different from other systems (WCDMA, CDMA2000, HSPA), e.g. channel definition, interferences, soft handoff. In general, soft handoff enforced by power control has been proposed as a possible solution to local traffic imbalances among cells [5]. Actually, power control is one of the most important processes, as interference is the predominant factor that affects the capacity and signal-to-interference ratio (SIR). To maximize system capacity, power control can be used efficiently to adapt cell sizes for load balancing; however, the trade-off between coverage and capacity should be carefully considered $[6,7]$. An adaptive load-shedding scheme combines the power control and the soft handoff function to force some mobile stations (MSs) farthest from the cell to enter forced soft handoff, and transfer their traffic load to neighboring cells that are lightly loaded. In this way, heavily loaded cells dynamically down-size their coverage area in order to handle traffic, while adjacent cells that are less heavily loaded increase their coverage to accommodate the extra traffic. However, in a hot-spot sector, powering up all MSs in the sector results in excessive interference with the MSs in neighboring cells, so they cannot maintain sufficient SIR levels at their sector sites. Previous studies also attempt to achieve constant received mean power from each MS within a sector [8-10].

This paper investigates the load balancing problem in an environment of heterogeneous traffic distributions. An analytical model is proposed to determine the effect of heterogeneous traffic distributions on load balancing, in which a generic measure of load balancing level (LBL) that is a function of traffic type coefficient (TYC) and call blocking probability of cells is to analyze the expected level of the load balance. We consider both voice traffic and data traffic to determine which kind of traffic has the greater effect. The performance of cellular systems with sectorization is evaluated; they are normal case $(\mathrm{N})$ of homogeneous distribution and linear case $(\mathrm{L})$ of heterogeneous distribution.

The remainder of this paper is organized as follows. In Section 2, we discuss the mobile cellular system background. In Section 3, we present an analytical model of load balancing, and define SIR. Section 4 details the numerical results, and Section 5 contains some concluding remarks.

\section{Background of Mobile Cellular Systems}

\section{$2.1 \quad$ Sectorization}

Generally speaking, a BS configuration is uniformly sectorized in one sector (with omnidirectional antenna, $360^{\circ}$ per sector), in three sectors $\left(120^{\circ}\right.$ per sector $)$, and in six sectors $\left(60^{\circ}\right.$ per sector). The capacity of each sector is calculated subject to the system's SIR requirements. A sector that is lightly loaded usually experiences more interference than a heavily loaded sector, which leads to a higher blocking probability in the lightly loaded sector. Denote $B$ as a set of BSs, and $S$ as a set of sectors configured in the BS. We further denote the sector $s$ in BS $j$ as sector $_{j s}(\forall s \in S, j \in B)$ and $K$ as the set of sector configurations. In this paper, the following two probable configurations are given for a BS $(|K|=2)$ : a single sector configuration with an omni-directional antenna, $\left(360^{\circ}\right.$ per sector $)$, and a three-sector configuration $\left(120^{\circ}\right.$ per sector $) ; k$ is assigned as the identification (ID) for each configuration. The sector ID $i$ identifies the sector in the configuration $k$ in an anti-clockwise direction. Table 1 summarizes the sector candidates 
Table 1: Sector candidates for the BS

\begin{tabular}{|c|c|c|c|}
\hline The value of $S$ & Candidate $s_{k, i}$ & Configuration I.D. $(k)$ & Sector I.D. $(i)$ \\
\hline$S=1$ & $S(1,1)$ & 1 & 1 \\
\hline$S=2$ & $S(2,1)$ & 2 & 1 \\
\hline$S=3$ & $S(2,2)$ & 2 & 2 \\
\hline$S=4$ & $S(2,3)$ & 2 & 3 \\
\hline
\end{tabular}

Table 2: Coverage of candidate sectors

\begin{tabular}{ccc}
\hline Candidate $S_{k, i}$ & Sector I.D. $i$ & Coverage of $S_{k, i}$ \\
\hline 1 & 1 & $\left(\phi, \phi+360^{\circ}\right)$ \\
2 & 1 & $\left(\phi, \phi+120^{\circ}\right)$ \\
3 & 2 & $\left(\phi+120^{\circ}, \phi+240^{\circ}\right)$ \\
4 & 3 & $\left(\phi+240^{\circ}, \phi+360^{\circ}\right)$ \\
\hline
\end{tabular}

for each BS for a combination of $k$ and $i$. Let $S$ be the set of sectors; then, each sector $s_{k, i}$ $\left(\forall s_{k, i} \in S\right)$ is defined by the sector configuration $(k)$ and the sector ID $(i)$. Table 2 details the coverage (in degrees) of each sector, where $\phi$ is the degree of the baseline. In general, it can be assigned arbitrarily, but in this paper we given $-30^{\circ}$ in our cellular structure example, as shown in Figure 1.

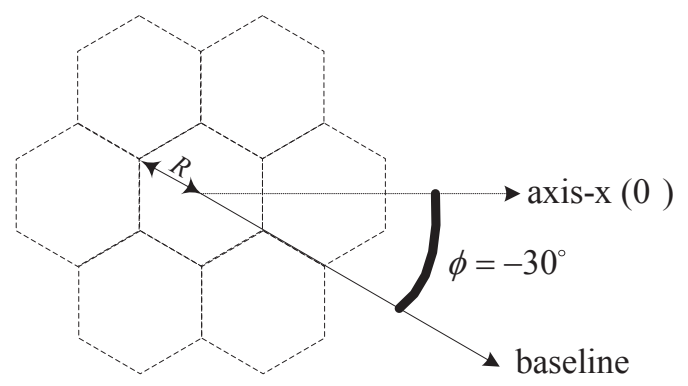

Figure 1: The baseline deployed in a cellular structure

\subsection{Interference between sectors}

To calculate the interference between sectors, the sector configuration information in Table 1 and the sector coverage information in Table 2 must be given. Without loss of generality, sector $s_{k, i}\left(s_{k \prime, i l}\right)$ is replaced by $s(s \prime)$; and sector $s$ in BS $j$ is denoted by sector $j s$, as shown in Figure 2. Because of the baseline degree deployed in all cells is the same using $\phi=-30^{\circ}$, no matter what the BS is configured, the mutual interference between sectors can be well-known. If we define the interference indicator functions $\Omega_{j s j / s l}^{U L}$ and $\Omega_{j s j / s !}^{D L}$ for the respective uplink $(U L)$ and downlink $(D L)$ connections between sector ${ }_{j s}$ and sector $_{j / s}$, they can be pre-calculated. To pre-calculate the indicator functions, the sector candidates to be configured in the BS must be defined. Assuming $\left(x_{j s}, y_{j s}\right)$ and $\left(x_{j / s}, y_{j / s l}\right)$ are the respective locations of BS $j$ and BS $j \prime$, the vectors $\overrightarrow{A_{1}}\left(\overrightarrow{A_{1}}\right)$ and $\overrightarrow{A_{2}}\left(\overrightarrow{A_{2}}\right)$ covering sector ${ }_{j s}\left(\right.$ sector $\left._{j / s l}\right)$ are defined as follows:

$$
\begin{gathered}
\overrightarrow{A_{1}}=\left[x_{j s}^{1}-x_{j s}, y_{j s}^{1}-y_{j s}\right], \overrightarrow{A_{2}}=\left[x_{j s}^{2}-x_{j s}, y_{j s}^{2}-y_{j s}\right] \\
\overrightarrow{A_{1}}=\left[x_{j \prime s \prime}^{1}-x_{j \prime s \prime}, y_{j \prime s \prime}^{1}-y_{j \prime s \prime}\right], \overrightarrow{A_{2}}=\left[x_{j \prime s \prime}^{2}-x_{j \prime s \prime}, y_{j / s \prime}^{2}-y_{j \prime s \prime}\right]
\end{gathered}
$$




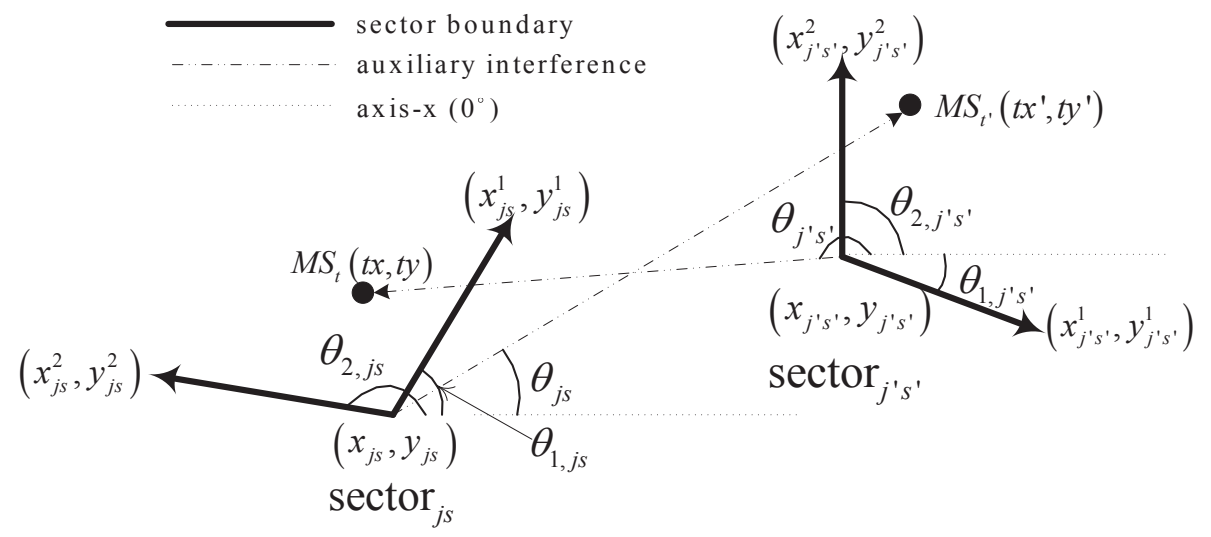

Figure 2: Mutual interference between sectors

where

$$
\begin{gathered}
x_{j s}^{1}=R_{j} \cos \left(\theta_{1, j s}\right)+x_{j s}, y_{j s}^{1}=R_{j} \sin \left(\theta_{1, j s}\right)+y_{j s}, \\
x_{j s}^{2}=R_{j} \cos \left(\theta_{2, j s}\right)+x_{j s}, y_{j s}^{2}=R_{j} \sin \left(\theta_{2, j s}\right)+y_{j s} \\
x_{j \prime s \prime}^{1}=R_{j \prime} \cos \left(\theta_{1, j \prime s \prime}\right)+x_{j \prime s \prime}, y_{j \prime s \prime}^{1}=R_{j \prime} \sin \left(\theta_{1, j / s \prime}\right)+y_{j \prime s \prime}, \\
x_{j \prime s \prime}^{2}=R_{j \prime} \cos \left(\theta_{2, j \prime s \prime}\right)+x_{j \prime s \prime}, y_{j \prime s \prime}^{2}=R_{j \prime} \sin \left(\theta_{2, j / s \prime}\right)+y_{j \prime s \prime}
\end{gathered}
$$

Then, the DL interference $\Omega_{j s j / s /}^{D L}$, between sector $_{j s}$ and sector ${ }_{j / s \prime}$ can be analyzed by an auxiliary vector $\overrightarrow{A_{3}}=\left[t x \prime-x_{j s}, \mathrm{t} y \prime-y_{j s}\right]$, where $(t x \prime, t y \prime)$ is the arbitrary position of MS $t \prime(\forall t \prime \in T$ and $T$ is the set of MSs) serviced by sector ${ }_{j \prime s /}$. Furthermore, $\left(\theta_{1, j s}, \theta_{2, j s}\right)$ and $\left(\theta_{1, j / s l}, \theta_{2, j / s l}\right)$ can also be calculated easily. According to $\overrightarrow{A_{3}}, \theta_{j s}$ is calculated by $\theta_{j s}=\tan ^{-1} \frac{t y \prime-y_{j s}}{t x /-x_{j s}}, 0 \leq \theta_{j s}<360^{\circ}$.

After calculating $\theta_{j s}$, the Algorithm Cal_InterF ${ }^{2}$ is applied to calculate $\Omega_{j s j / s}^{D L}$ Meanwhile, the UL interference $\Omega_{j s j / s !}^{U L}$ between sector ${ }_{j s}$ and sector ${ }_{j / s l}$ can be analyzed by an auxiliary vector $\overrightarrow{A_{3}}=\left[t x-x_{j / s l}\right.$, ty $\left.-y_{j / s l}\right]$, where $(t x, t y)$ is the arbitrary position of MS $t(\forall t \in T)$ serviced by sector $_{j s} ; \theta_{j / s \prime}$ is calculated by $\theta_{j / s \prime}=\tan ^{-1} \frac{t y-y_{j / s /}}{t x-x_{j / s} \prime}, 0 \leq \theta_{j / s \prime}<360^{\circ}$. Again, applying Algorithm Cal_InterF to the calculation of UL interference $\Omega_{j s j / s /}^{U L}$.

\section{Analytical Model of Load Balancing}

\subsection{SIR definition}

Denote $z_{j s t}$ as a decision variable, which is 1 if MS $t$ is admitted by sector ${ }_{j s}$ subject to the SIR requirements and 0 otherwise. Assuming the power of both the UL and DL are perfectly controlled, the received power in sector ${ }_{j s}$ from MS $t$ with constant value $P_{c(t)}^{U L}$ will be in the same traffic class $-c(t)$ in the UL, and the received power at MS $t$ from sector ${ }_{j s}$ with constant value $P_{c(t)}^{D L}$ will be in same traffic class $-c(t)$ in the DL. If $D_{j t}$ is the distance from MS $t$ to sector ${ }_{j s}$, and given an attenuation factor $\tau=4$ which is the degree to which a beam of radiation has been attenuated, the intra-sector interference on the UL and the DL is given by (1) and (2) respectively, where both $\alpha_{c(t)}^{U L}$ and $\alpha_{c(t)}^{D L}$ are activity factors of traffic class- $-c(t)$. The inter-sector

\footnotetext{
${ }^{2}$ Detailed algorithm procedure is omitted due to the length limitation of the paper. A complete version of the procedure is available upon request.
} 
interference on the UL and the DL is expressed by (3) and (4) respectively, where both $\Omega_{j / s}^{U L}$ s and $\Omega_{j / s / j s}^{D L}$ indicate the interference between sectors.

$$
\begin{aligned}
& I_{j s t, i n t r a}^{U L}=\sum_{\substack{t \prime \in T \\
t / \neq t}} \alpha_{c(t \prime)}^{U L} P_{c(t \prime)}^{U L} z_{j s t \prime} \\
& I_{j s t, i n t r a}^{D L}=\sum_{\substack{t \prime \in T \\
t \prime \neq t}} \alpha_{c(t \prime)}^{D L} P_{c(t \prime)}^{D L}\left(\frac{D_{j t \prime}}{D_{j t}}\right)^{\tau} z_{j s t \prime} \\
& I_{j s t, i n t e r}^{U L}=\sum_{\substack{j \prime \in B \\
j \neq 1}} \sum_{\substack{s / \in S \\
s / \neq s}} \sum_{\substack{t / \in T \\
t / \neq t}} \Omega_{j \prime s / j s}^{U L} \alpha_{c(t \prime)}^{U L} P_{c(t \prime)}^{U L}\left(\frac{D_{j \prime t \prime}}{D_{j t \prime}}\right)^{\tau} z_{j \prime s / t \prime} \\
& I_{j s t, i n t e r}^{D L}=\sum_{\substack{j \prime \in B \\
j \neq j}} \sum_{\substack{s / \in S \\
s / \neq s}} \sum_{\substack{t \prime \in T \\
t / \neq t}} \Omega_{j \prime s / j s}^{D L} \alpha_{c(t \prime)}^{D L} P_{c(t \prime)}^{D L}\left(\frac{D_{j \prime t \prime}}{D_{j / t}}\right)^{\tau} z_{j \prime s / t \prime} \\
& S I R_{j s, c(t)}^{U L}=\frac{W^{U L}}{d_{c(t)}^{U L}} \cdot \frac{P_{c(t)}^{U L}+\left(1-z_{j s t}\right) V}{\left(1-\rho^{U L}\right) I_{j s t, \text { intra }}^{U L}+I_{j s t, \text { inter }}^{U L}} \\
& S I R_{j s, c(t)}^{D L}=\frac{W^{D L}}{d_{c(t)}^{D L}} \cdot \frac{P_{c(t)}^{D L}+\left(1-z_{j s t}\right) V}{\left(1-\rho^{D L}\right) I_{j s t, \text { intra }}^{D L}+I_{j s t, \text { inter }}^{D L}}
\end{aligned}
$$

Let $W^{U L}\left(W^{D L}\right)$ be the spectrum allocated to the UL (DL), and $d_{c(t)}^{U L}\left(d_{c(t)}^{D L}\right)$ be the information rate in the UL (DL). The SIR values $S I R_{j s, c(t)}^{U L}$ and $S I R_{j s, c(t)}^{D L}$ in the UL and the DL are defined in (5) and (6) respectively, where $\rho^{U L}\left(\rho^{D L}\right)$ is the UL (DL) orthogonality factor. Equations (5) and (6) give a very large artificial constant value $V$ in the numerator in order to satisfy the SIR constraints. This is because the SIR value must be larger than a pre-defined threshold, say the bit energy to noise ratio (BENR), if MS $t$ is to be admitted by $\operatorname{sector}_{j s}\left(z_{j s t}=1\right)$; in other words, the constraint BENR $\leqq$ SIR must be satisfied. For example, in the UL in Equation (5), if MS $t$ is to be admitted by $\operatorname{sector}_{j s}\left(z_{j s t}=1\right)$, the SIR value $S I R_{j s, c(t)}^{U L}$ is calculated by $\left(W^{U L} / d_{c(t)}^{U L}\right) \cdot P_{c(t)}^{U L} /\left(\left(1-\rho^{U L}\right) I_{j s t, \text { intra }}^{U L}+I_{j s t, \text { inter }}^{U L}\right)$ to determine whether the SIR constraint can be satisfied. In contrast, if MS $t\left(z_{j s t}=0\right)$ is rejected, the SIR value is always larger than BENR (BENR «SIR) because the value $V$ is dominant $P_{c(t)}^{U L}$; thus, $S I R_{j s, c(t)}^{U L}$ is calculated as a very large value. This implies that the constraint BENR $\leqq$ SIR can be ignored, as it is always satisfied.

\subsection{The Analytical Model}

In this paper, we consider traffic with multiple classes, and use the Kaufman model [12] as a performance measure to analyze the blocking probability of each traffic class effectively. Assume that $\mathrm{M}$ channels are shared by all traffic requirements. Then, for each traffic class $-c(\forall c \in C)$ with distinct channel requirements, the traffic arrival is a stationary Poisson process with mean rate $\lambda$; and the channel requirement $b$ is an arbitrary discrete random variable $\left(\operatorname{Prob}\left\{b=b_{c}\right\}=\right.$ $\left.q_{c}, \forall c \in C\right)$. A call request with channel requirement $b_{c}$ has a mean holding time of $1 / \mu_{c}$. Thus, traffic with channel requirement $b_{c}$ is generated in the Poisson arrival process with mean rate $\lambda_{c}=\lambda q_{c}$ and the class $-c$ offered load $a_{c}=\lambda_{c} / \mu_{c}$. The blocking probability of traffic class $-c$ is defined in (7) [12], where the distribution of $q(\cdot)$, which is the probability of the total number of channels occupied by the complete sharing policy, satisfies Equation (8) [11], and $q(x)=0$ for $x<0$ and $\sum_{j=0}^{M} q(x)=1$.

$$
B^{c}(a, b)=\sum_{i=0}^{b_{c}-1} q(|M|-i) \quad \forall c \in C
$$




$$
\sum_{c \in C} a_{c} b_{c} q\left(j-b_{c}\right)=j q(j) \quad j=0,1, \ldots, M
$$

To deal with variations in the traffic load, we can seek load balancing with an average value of resource utilization [12]. In order to evaluate the experiment results, we define a diversity function for system load balancing, from which the standard deviation (SD) of the call blocking probability among sectors can be derived. The smaller the SD, the better the balancing results will be. Let $g_{j s}^{c}=\sum_{t \in T} z_{j s t} / \mu_{c(t)}$ be the traffic intensity of class $-c$. Then, $g_{j s}=\sum_{c \in C} g_{j s}^{c}$ is the aggregate traffic (in Erlangs) in sector $j_{s}$, where $g_{j s}$ is equivalent to the traffic load $a$ in (7). We also define $m_{j s}=\sum_{t \in T} z_{j s t} m^{c(t)}$ as the total number of channels allocated in sector ${ }_{j s}$, where $m_{j s}$ is equivalent to the required channels $b$ in $(7)$, and $m^{c(t)}$ is the number of channels required for traffic class $-c(t)$. The performance measure $B_{j s}^{c}$ (the call blocking probability of traffic class $-c$ in sector $_{j s}$ ) is expressed by (7), where the sub-script $j s$ in $B_{j s}$ indicates $B^{c}$ in sector ${ }_{j s}$. If we define $L_{L B}$ as the LBL, the load balancing model can be formulated as (9), where $S D\left(B_{j s}^{c}\right)$ is the SD function of $B_{j s}^{c}$. The model is calculated subject to SIR Constraints (5) and (6).

$$
L_{L B}=\sum_{j \in B} \sum_{s \in S} \sum_{c \in C} K^{c} S D\left(B_{j s}^{c}\right) \quad \forall j \in B, s \in S
$$

To assess the impact of different traffic types on load balancing, we denote $K^{c}$ as a ratio of traffic class $-c$, where $\sum_{c \in C} K^{c}=1 . K^{c}$ is a traffic type coefficient (TYC) used to analyze the expected level of the load balance. Given two classes of call requests, e.g., voice and data traffic, if $K^{v}=1$ and $K^{d}=0$, we only investigate the effect of voice traffic on load balancing; however, if $K^{v}=0$ and $K^{d}=1$, we only investigate the effect of data traffic on load balancing.

\section{Numerical Results}

\subsection{Parameters}

Two heterogeneous traffic distributions are considered in the structure of a $5 \times 5$ twodimensional array with hexagonal cells, and their impact on the system's load balance is compared with that of a homogeneous distribution between sectors, as shown in Figure 3. In the figure, each dark cell has an heterogeneous load that is either heavier or lighter than the load of the normal cells (the light cells). It is assumed that the user density in each cell is homogeneous. Each cell is configured with 3 sectors $(|S|=3)$, and assigned a radius $R_{j s}=5.0 \mathrm{~km}$. The required $\operatorname{BENR}$ for voice $(v)$ and data $(d)$ traffic is given by $\left(E_{b} / N_{T O T A L}\right)_{v}^{U L}=\left(E_{b} / N_{T O T A L}\right)_{v}^{D L}=$ $7 \mathrm{~dB}$ and $\left(E_{b} / N_{T O T A L}\right)_{v}^{D L}=\left(E_{b} / N_{T O T A L}\right)_{d}^{D L}=10 \mathrm{~dB}$ respectively [13]. The information rates $d_{v}^{U L}=d_{v}^{D L}=9.6 \mathrm{bps}, d_{d}^{U L}=19.2 \mathrm{bps}, d_{d}^{D L}=38.4 \mathrm{bps}[14-16]$, and the activity factors $\alpha_{v}^{U L}=\alpha_{v}^{D L}=\alpha_{d}^{U L}=\alpha_{d}^{D L}=0.5[13,16,17]$ are also given. The number of channels required is $m^{v}=1, m^{d}=4$, and the orthogonality factor is $\rho^{U L}=0.9, \rho^{D L}=0.7$ [13]; and the power is perfectly controlled by $P_{v}^{U L}=10 \mathrm{~dB}, P_{v}^{D L}=15 \mathrm{~dB}, P_{d}^{U L}=15 \mathrm{~dB}, P_{d}^{D L}=20 \mathrm{~dB}$. The assigned service rate is $\Phi_{j s}^{v}=\Phi_{j s}^{d}=0.1[17,18]$.

\subsection{Traffic Models}

For each sector, call requests for both voice and data calls are generated in the Poisson arrival process with $\lambda_{v}$ and $\lambda_{d}$ respectively. The mean call holding time is given as $1 / \mu_{v}=180$ (sec), $1 / \mu_{d}=600(\mathrm{sec})[18]$. Denote $\left(E_{b} / N_{T O T A L}\right)_{c(t)}^{U L} \leq S I R_{j s, c(t)}^{U L}$ and $\left(E_{b} / N_{T O T A L}\right)_{c(t)}^{D L} \leq S I R_{j s, c(t)}^{D L}$ as the QoS requirements of the UL and DL respectively. All traffic calculated in $g_{j s}$ must satisfy the QoS requirements and the condition $z_{j s t} D_{j t} \leq R_{j_{s}} \delta_{j s t}$, where $\delta_{j s t}$ is the indicator function if MS $t$ is in the coverage of sector $j_{s}$. Power is perfectly controlled in both the UL and the 


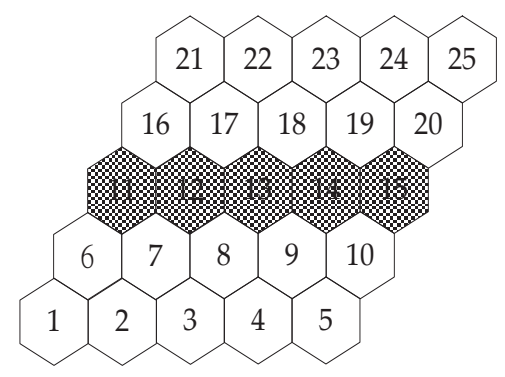

(a) Heterogeneous linear model

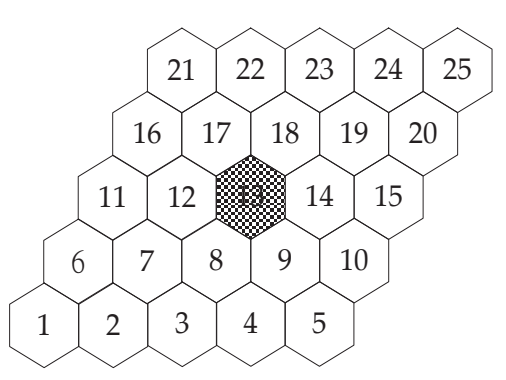

(b) Heterogeneous hot spot model

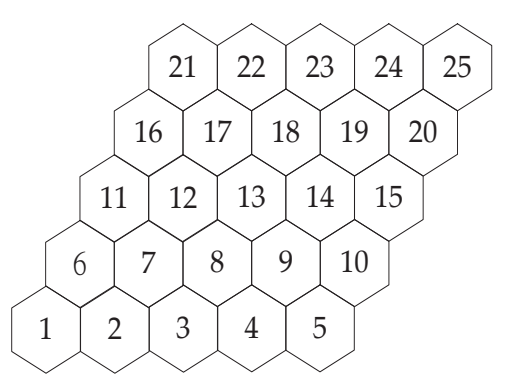

(c) Uniform model

Figure 3: Traffic distribution scenarios

DL, and soft handoff is not taken into account. The traffic distributions considered in this work are uniform $(\mathrm{U})$, hot spot $(\mathrm{H})$, and linear $(\mathrm{L})$, as shown in Figure 3. Recall that the cells with heterogeneous loads in Figure 3 (a) and Figure 3 (b) have either heavier or lighter loads than normal cells in a homogeneous distribution. To evaluate the heterogeneous scenario, we introduce two traffic models. We denote heterogeneous cells with heavier loads as M1 and heterogeneous cells with lighter loads as M2. If normal cells are given traffic arrivals $\lambda_{c}$ for traffic class $-c$, arrivals in heterogeneous cells are assigned $200 \%$ of $\lambda_{c}$ in M1, and $50 \%$ of $\lambda_{c}$ in M2. Thus, the level of load balance for traffic with multiple classes can be evaluated effectively in a near-realistic environment.

\subsection{Analysis}

Without loss of generality, the level of load balance is represented in logarithmic form $\log \left(F_{L B}\right)$. If a smaller value of $\log \left(F_{L B}\right)$ is calculated, a better level of load balance will be achieved. In Figure 4 (a), no matter what the distribution (linear, hot spot, or uniform) and the offered voice arrivals $\lambda_{v}$ are, $\log \left(F_{L B}\right)$ is a decreasing function of $K^{v}$. This implies that load balancing can be achieved more easily for voice only traffic than for data only traffic. If only data traffic is considered, given $K^{v}=0$ for all distributions, $\log \left(F_{L B}\right)$ is nearly -1.75 , whereas $\log \left(F_{L B}\right)$ is nearly -2.4 if only voice traffic is considered $\left(K^{v}=1\right)$. With regard to the effect of traffic intensity, it is easier to achieve load balancing with more offered voice traffic than less offered traffic. For example, in Figure 4 (a), given $K^{v}=0.5$ with $\lambda_{d}=6, \log \left(F_{L B}\right)$ calculates $(-1.2,-1.6,-1.9)$ for arrivals $\left(\lambda_{v}=12,30,48\right)$. Again, given $\lambda_{v}=12$ in Figure $4(\mathrm{a}), \log \left(F_{L B}\right)$ is in the range -1.15 to -1.4 in Figure 4 (a), but it is in the range -1.7 to -1.75 in Figure $4(\mathrm{~b})$ with $\lambda_{d}=24$.

For the traffic models (M1 vs. M2), there is no significant difference in the load balance with $\lambda_{v}=12$ and $\lambda_{d}=6$ in both Figure 4 (a) and Figure 5 (a). However, given $\lambda_{d}=6$ in Figure 5 (a), the load balance level varies in heavily loaded voice traffic $\left(\lambda_{v}=30,48\right)$. In another case, given $\lambda_{d}=24$ in Figure $5(\mathrm{~b}), \log \left(F_{L B}\right)$ calculates the same results for variations in the load balance. From the analysis, we conclude that the level of load balancing is more stable in M1 than in M2. A better scheme is needed to handle load balancing in cases of heterogeneous cells with light traffic loads.

\section{Conclusion}

In this paper, we propose a load balancing model to deal with the ever-increasing number of heterogeneous distributions in mobile wireless communication systems. We have studied the 


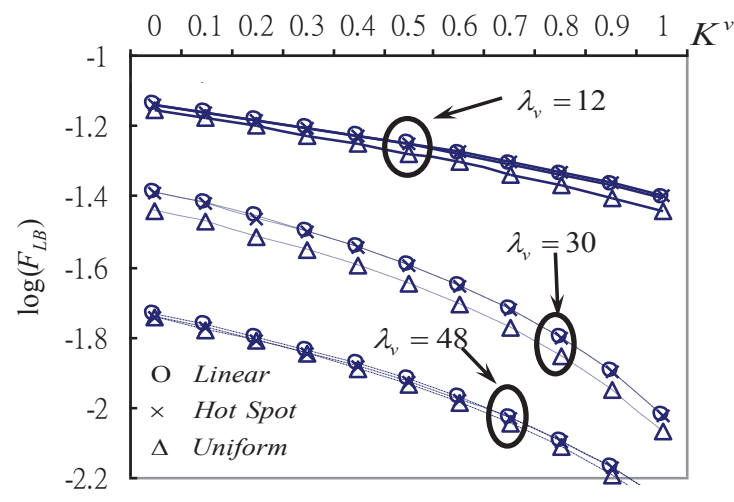

(a) $\lambda_{d}=6$

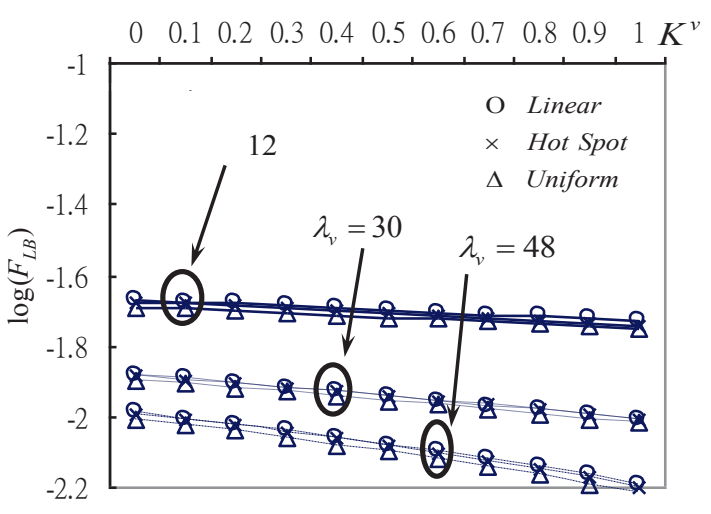

(b) $\lambda_{d}=24$

Figure 4: BLF as a function of BLC $K^{v}$ with respect to $\lambda_{v}$, given traffic model M1 and $|S|=3$

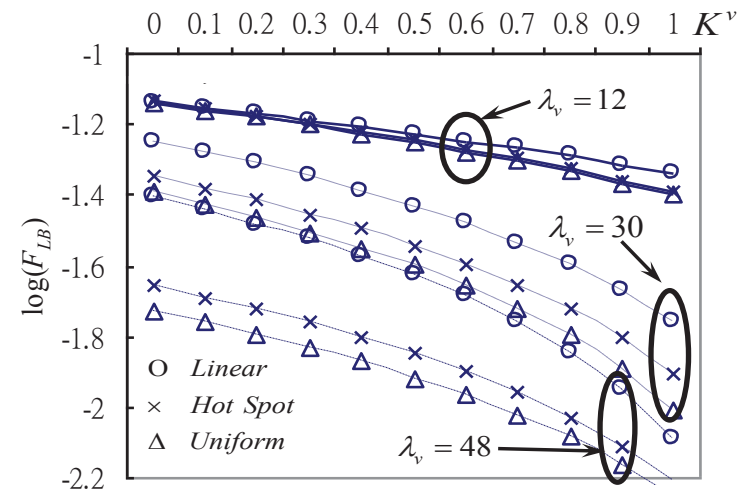

(a) $\lambda_{d}=6$

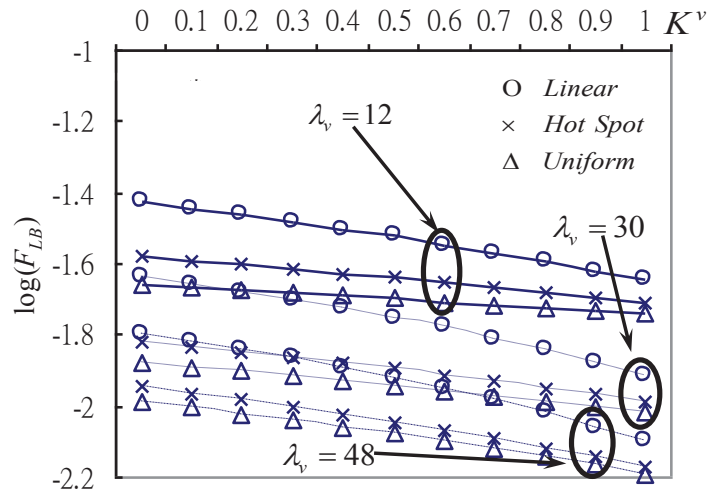

(b) $\lambda_{d}=24$

Figure 5: BLF as a function of BLC $K^{v}$ with respect to $\lambda_{v}$, given traffic model M2 and $|S|=3$

effect of heterogeneous traffic distributions on load balancing, as well as the effect of sectorization. The numerical results indicate that the level of load balancing is affected by spatial traffic distributions, especially by lighter loads in heterogeneous cells (the M2 model). In the scenario of heterogeneous cells with heavier loads (the M1 model), the level of load balancing has similar values of $\log \left(F_{L B}\right)$ in three distributions, i.e., the linear, hot spot, and uniform models. Sectorization is more effective in achieving load balancing in the scenario of the heavier loads than in the lighter loads. To achieve load balancing as well as capacity maximization in a system with heterogeneous distributions, a hybrid FMDA/CDMA scheme can be utilized. Usually, available wideband spectrum can be divided into a number of subspectra with smaller bandwidths; each of them is further deployed by CDMA technique. Each subspectrum employs direct sequence spectrum spreading with reduced processing gain, which is transmitted in one and only one subspectrum. The scheme moderately mitigates interference by allocating an appropriate subspectrum in each cell. The results of this work are useful for network planning to optimize the channel allocation for different traffic type's distribution. 


\section{Bibliography}

[1] J.-S. Wu and J.-K. Chung, Analysis of uplink and downlink capacities for two-tier cellular system, IEEE Proc.-Communications., 144(6):405-411, 1997

[2] J.C. Liberti, T. S. Rappaport, Analytical Results for Capacity Improvements in CDMA, IEEE Transactions on Vehicular Technology, vol. 43, pp. 680-690, Sep. 1994

[3] G. Ning, G. Zhu, L. Peng, and X. Lu, Research on hybrid dynamic load balancing algorithm in heterogeneous hierarchical wireless networks, Journal on Communication, 28(1):75-81+86, 2007

[4] A. Sang, X. Wang, M. Madihian, and R. D. Gitlin, Coordinated load balancing, handoff/cellsite selection, and scheduling in multi-cell packet data systems, Wireless Networks, 14(1):103120,2008

[5] X.H. Chen, Adaptive traffic-load shedding and its capacity gain in CDMA cellular systems, in Proc. IEE Communications, pp. 186-192, 1995

[6] V.V. Veeravalli and A. Sendonaris, The coverage-capacity tradeoff in cellular CDMA systems, IEEE Transactions on Vehicular Technology, 48(5):1443-1450, 1999

[7] G. Hampel, K.L. Clarkson, J.D. Hobby, and P.A. Polakos, The tradeoff between coverage and capacity in dynamic optimization of $3 \mathrm{G}$ cellular networks, in Proc. IEEE VTC-Fall, vol. 2, pp. 927-932, 2003

[8] W.-M. Tam and F.C.M. Lau, Analysis of power control and its imperfections in CDMA cellular systems, IEEE Transactions on Vehicular Technology, 48(5):1706-1717, 1999

[9] X.H. Chen and K.L. Lee, A novel adaptive traffic load shedding scheme for CDMA cellular mobile systems, in Proc. IEEE ICCS, vol. 2, pp. 566-570, 1994

[10] X.H. Chen, Adaptive traffic-load shedding and its capacity gain in CDMA cellular mobile systems, IEE Proceedings-Communications, 142(3):186-192, 1995

[11] J.S. Kaufman, Blocking in a shared resource environment, IEEE Transactions on Communications, vol. 29, pp. 1474-1481, 1981

[12] A. He, Performance comparison of load balancing methods in multiple carrier CDMA systems, in Proc. IEEE PIMRC, pp. 113-118, 2000

[13] S.W. Kim, D.G. Jeong, W.S. Jeon, and C.-H. Choi, Forward link performance of combined soft and hard handoff in multimedia CDMA systems, IEICE Transactions on Communications, vol. E85-B, no.7, pp. 1276-1282, July 2002

[14] W. Choi and J.Y. Kim, Forward-link capacity of a DS-CDMA system with mixed multirate sources, IEEE Transactions on Vehicular Technology, 50(3):737-749, 2001

[15] D. Kim and D.G. Jeong, Capacity unbalance between uplink and downlink in spectrally overlaid narrow-band and wide-band CDMA mobile systems, IEEE Transactions on Vehicular Technology, 49(4):1086-1093, 2000

[16] W.S. Jeon and D.G. Jeong, Call admission control for mobile multimedia communications with traffic asymmetry between uplink and downlink, IEEE Transactions on Vehicular Technology, 50(1):59-66, 2001 
[17] W.S. Jeon and D.G. Jeong, Call admission control for CDMA mobile communications systems supporting multimedia services, IEEE Transactions on Wireless Communications, 1(4):649-659, 2002

[18] K. Kim and Y. Han, A call admission control scheme for multi-rate traffic based on total received power, IEICE Transactions on Communications, vol. E84-B, no. 3, pp. 457-463, March 2001 